\title{
Research on Carbon Emission Management System Deployed in Civil Airport
}

\author{
Bo $\mathrm{Li}^{1,2}$, Wen Zhang ${ }^{1,2, *}$, Ran $\mathrm{Lei}^{3}$ \\ ${ }^{1}$ China Airport Construction Group Co., Ltd, 100101, China \\ ${ }^{2}$ Beijing Super-Creative Technology Co., Ltd, 100621, China \\ ${ }^{3}$ Beijing Daxing International Airport, 102602, China
}

\begin{abstract}
Along with the increasingly prosperous airport industry, energy consumption and carbon emission at airport rocket, rendering the building of eco-friendly airports becoming the common trend and objective of global airport development. Digging into airport carbon emission management is of relevance for reducing airport carbon emission and improving the quality of airports and surrounding environment. The article analyzes the needs of airport carbon emission management given the current situation, proposes to achieve airport carbon emission management and monitoring by using information technology, develops a carbon management system software for civil aviation airports, and provides smart strategies for airport carbon emission management.
\end{abstract}

\section{Overview}

The rapid progress made in civil aviation infrastructure benefits the whole world, and airports, as the major infrastructure, inevitably leave more "carbon footprint". By calculation, the global air transport industry contributes about $2 \%$ of the global total carbon emission, in which airport activities take up about 5\%. As people concern more about carbon emission reduction, ecofriendly airports have become the common goal of global airport development. Some countries, regions and international organizations have introduced carbon emission reduction regulations and programs: The European Union announced the plan to subject airlines to the management of the Emissions Trading Scheme (ETS) in September 2005; In 2008, the Civil Aviation Administration of China promulgated the Planning on Energy Conservation and Emission Reduction of Civil Aviation Industry, requiring the whole industry to vigorously promote aviation energy conservation and emission reduction; In 2011, the Guiding Opinions on Accelerating the Energy Conservation and Emission Reduction of Civil Aviation Industry was issued, in which propositions were made that the growth rate of energy consumption and $\mathrm{CO}_{2}$ emission of the whole industry must be lower than the development rate of the industry, and by 2020, the energy consumption and emission of China's civil aviation per unit of output decrease by $22 \%$ compared with that of 2005. In 2017, China launched the carbon trading market. The civil aviation and six other industries were chosen as the first batch for pilot.

In the future, with the tightening of China's environmental policies, the continuous expansion of civil airports in both number and scale and the complexity of large-scale airports, energy conservation and emission reduction will expose the airport industry to challenges by letting it bear the burden of "paying carbon tax" internationally and the pressure of "developing green civil aviation" domestically.

\section{Demand Analysis of Airport Carbon Emission Management}

Aiming to promote the construction of eco-friendly airports, the Civil Aviation Administration issues a series of policy documents such as the Implementation Opinions on Deepening the Green Development of Civil Aviation, further clarifying the objectives and tasks of the green development of civil aviation airports. At present, the construction of airports that are safe, green, intelligent and people-centered is in full swing. However, subpar carbon emission reduction standards, obsolescence in the development of energy consumption and carbon emission calculation and statistics systems and the gap in effective management methods are ailing domestic airports. The looming pressure of carbon emission reduction requires giving top priority to the study of the operation-generated. carbon emission volume and emission characteristics, which is the premise for future implementation and evaluation of airport carbon emission reduction.

Under this circumstance, the establishment of an airport carbon emission management system is an effective way to promote energy conservation and emission reduction in the airport industry, and is also a necessary means to deal with domestic control and development of the airport low-carbon economy. The airport carbon emission management system, running on accurate carbon emission data, can effectively bolster

*Corresponding author: zhangwen313@qq.com 
airports for carbon emission reduction, facilitate the detail understanding of carbon emission actuality, evaluate the outcome of carbon emission reduction measures already implemented, and provide basis and support for formulating energy conservation and emission reduction measures.
Based on the above considerations per the carbon emission system demands, the contents of each module and the system flow logic are sorted out from the system function module level, as shown in the following figure.

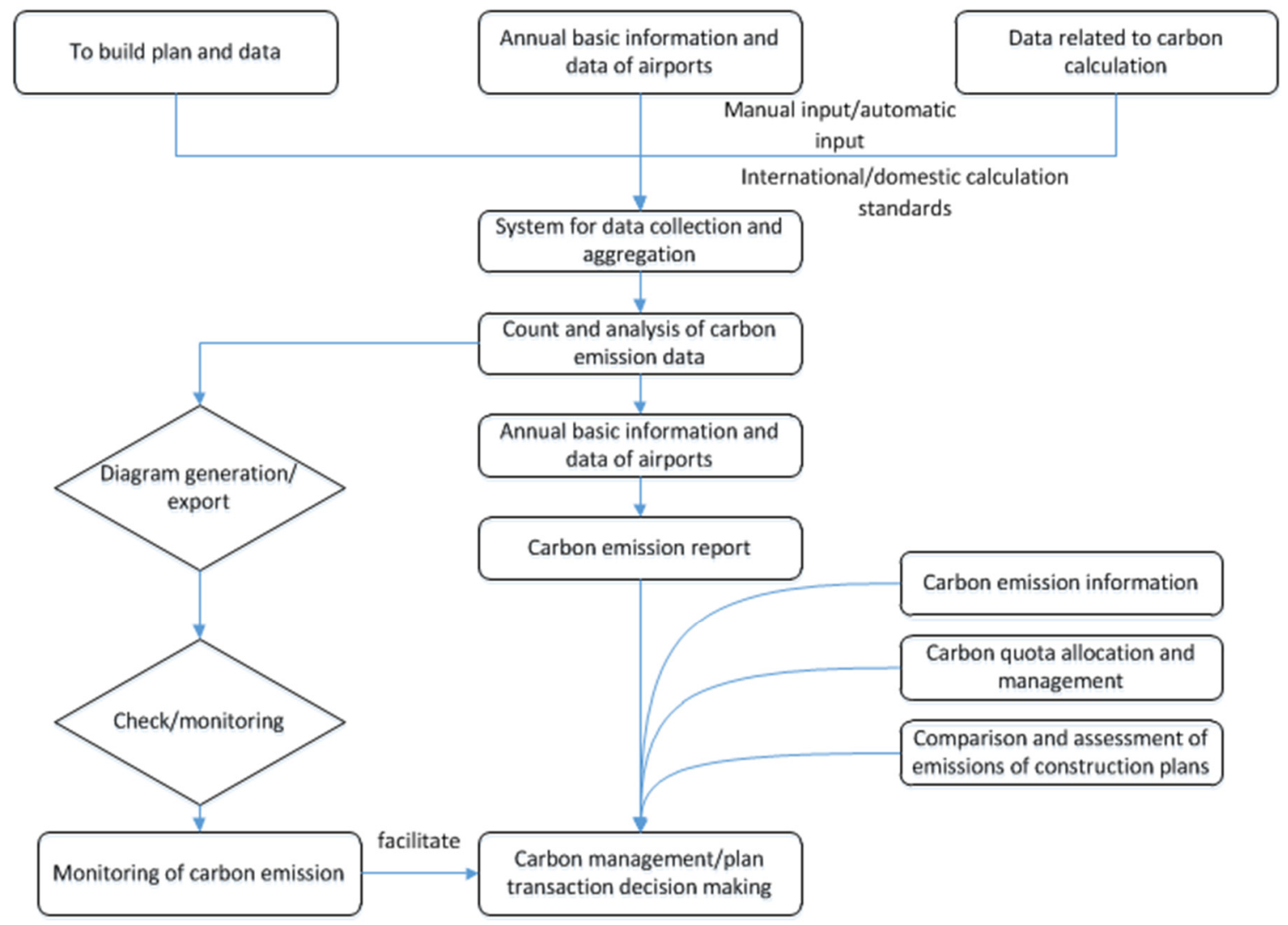

Figure 1. Route Chart of System Technology

\section{Architecture and Basic Functions of Airport Carbon Emission Management System}

\subsection{Airport Carbon Emission Management System Architecture}

The airport carbon emission management system applies the SaaS software under the $\mathrm{B} / \mathrm{S}$ framework and the system architecture is divided into different levels, i.e. the operating system, the database, the module processing, the software application and the browsing. Via internet browser, services are provided for airport carbon emission management system users. The system supports the of data and configuration between different users to ensure the security and privacy of each user data, as well as reserve space for tailored demands of future users such as interfaces, business logic, and data structures.

Three types of people with different usage authorities are the target users of the system: system administrators, system data entry personnel and people viewing/analyzing system data (such as the airport party, management leaders, etc.). For example, the administrator account has all the authorities, able to add/edit accounts of the data entry party and the airport party to ensure the normal operation of the system and allow multiple parties to manage and analyze the carbon data reasonably; the account authorities obtained by the airport party are limited to data viewing and exporting partial data and reports; the data entry party can obtain the accounts with data entry authorities to edit and import the platform data (data source: energy system related data or data submitted by organizations) to ensure data security and accuracy. Administrators can set up accounts with different authorities and hand them to relevant personnel who can verify and enter (or import from similar energy data systems according to data and units required in national standards) the basic data of the carbon management system (such as basic information of airports, emission boundaries, emission sources, emission factors and emission data). The airport party or the construction party can then check the authenticity and validity of the data. After the verification process, the system can collect different types of data according to different categories, calculate the final carbon emission data and display the basic data chart of each data, and simultaneously generate data statements of different fields highlighted in the carbon management. As it is shown in Figure 2. 


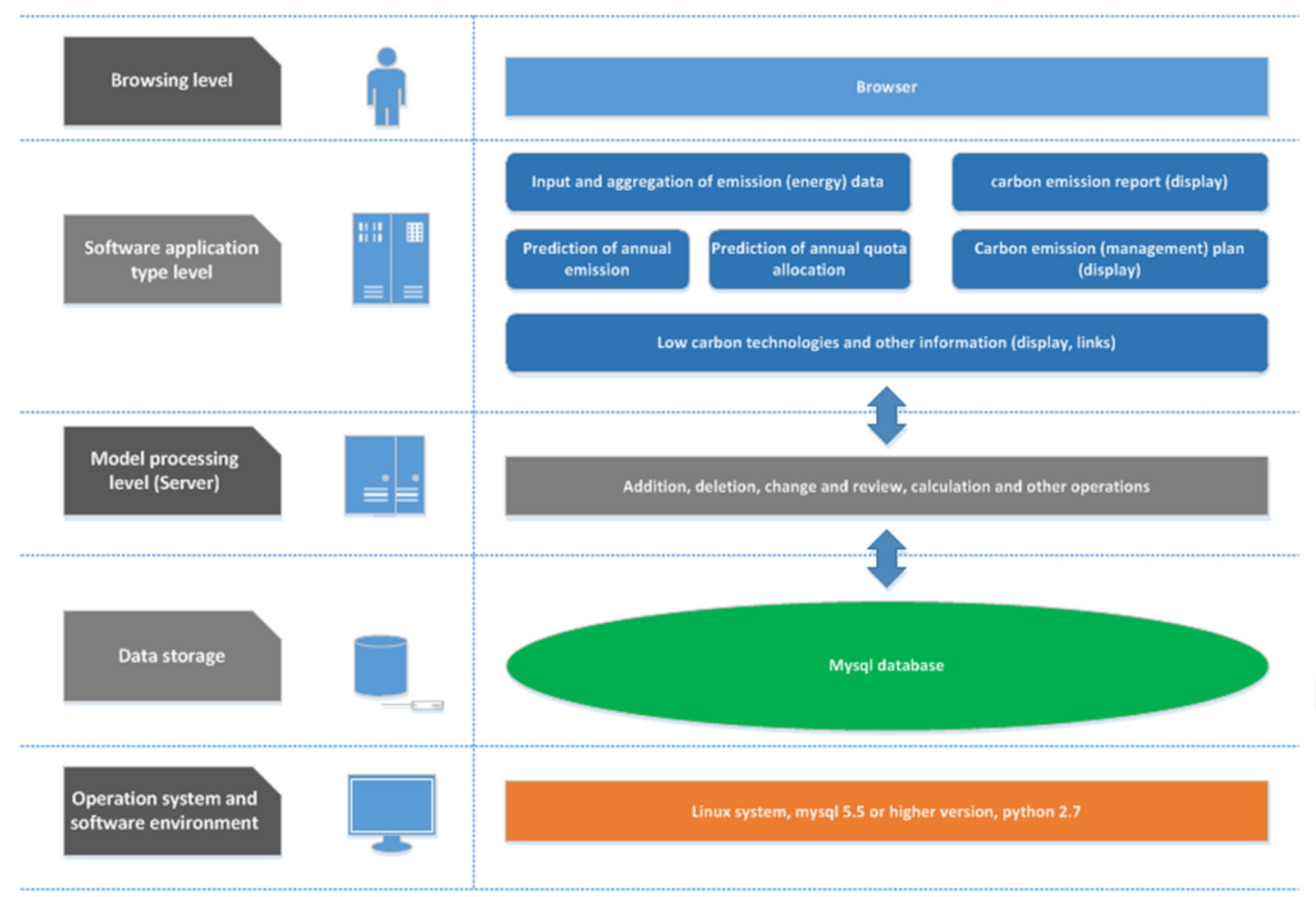

Figure 2. Architecture of carbon emission system

\subsection{Main functions realized by the system}

The system functions mainly include airport information and carbon emission boundary management, carbon emission data collection and aggregation, carbon emission data analysis, carbon emission report generation, carbon management method selection, carbon quota allocation forecasting and management, carbon emission comparison and evaluation of project construction plan and multi-level login/management modules.

\subsubsection{To achieve carbon emission monitoring and management during the airport operation process}

The module can collect data on various carbon emission activities of the airport according to international/national authoritative standards: Through the network transmission, scattered front-end carbon emission data information of each part of the airport can be imported and automatically entered into the system per category, providing basic data guarantee for calculating the greenhouse gas emissions for the future, obtaining carbon dioxide equivalent $\left(\mathrm{CO}_{2} \mathrm{e}\right)$ data, improving the scientific and comprehensive carbon management data and bolstering future carbon management efficiency. Realtime online browsing (category selection and browsing) can be enabled simultaneously to comprehensively view and grasp the carbon emission of airports so as to achieve online monitoring and management of airport carbon emissions.

\subsubsection{To realize the statistics and analysis of airport carbon emissions data}

Automatic analysis of airport carbon emission data and automatic generation of carbon emission reports will be provided to lay decision-making foundation for the evaluation of emission reduction measures and the formulation of energy conservation and emission reduction measures.

The system can sort and analyze the collected airport carbon emission data, calculate the final emission volume of all greenhouse gases through the standardized platform module, and summarize the emissions of various dimensions in the year for in-depth analysis and comparison carbon emissions data mining, and the generation of analysis charts of different classification and different dimensions. Then, users can more intuitively and thoroughly understand the airport's carbon emission, and the decision-making basis for the evaluation of emission reduction measures and the formulation of energy-saving and emission reduction measures can be also secured. As it is shown in Figure 3.

In addition, the system enables automatic generation of carbon emission reports. The collected raw data and the results of the statistical analysis are organized into a document that is easy for administrators to review according to a certain format (for example, the existing inventory report standard), and the generated list and carbon emission report can be downloaded. Users may edit the template of the data list through the system module, organize the ideas of carbon emission report, and finally retain the ideas by generating a template to provide 
basic ideas for future carbon management. In the future, users are also empowered to freely define and edit new templates and report templates to be generated according to policies, laws and regulations or other influencing factors. Users may discuss the composition of the report with administrators and determine the final plan. Lists and reports will be output in PDF or Excel format, taking into account the stability of the browsing format.

\subsubsection{To accurate predict carbon trading quota}

The system can automatically calculate and predict the basic carbon quota according to the quota allocation plan set by the state by analyzing and calculating the historical carbon emission data of the airport and emission data specified by major emitting organizations, and comparing the results with those of other similar airports. Through the analysis of the estimated quota and historical data, a suitable method for managing carbon quota can be found to lay a solid foundation for the full tap into the carbon trading market in the future. The system interface will be also reserved for improving the module in the future according to the actual situation to accommodate the real, more complex trading market.

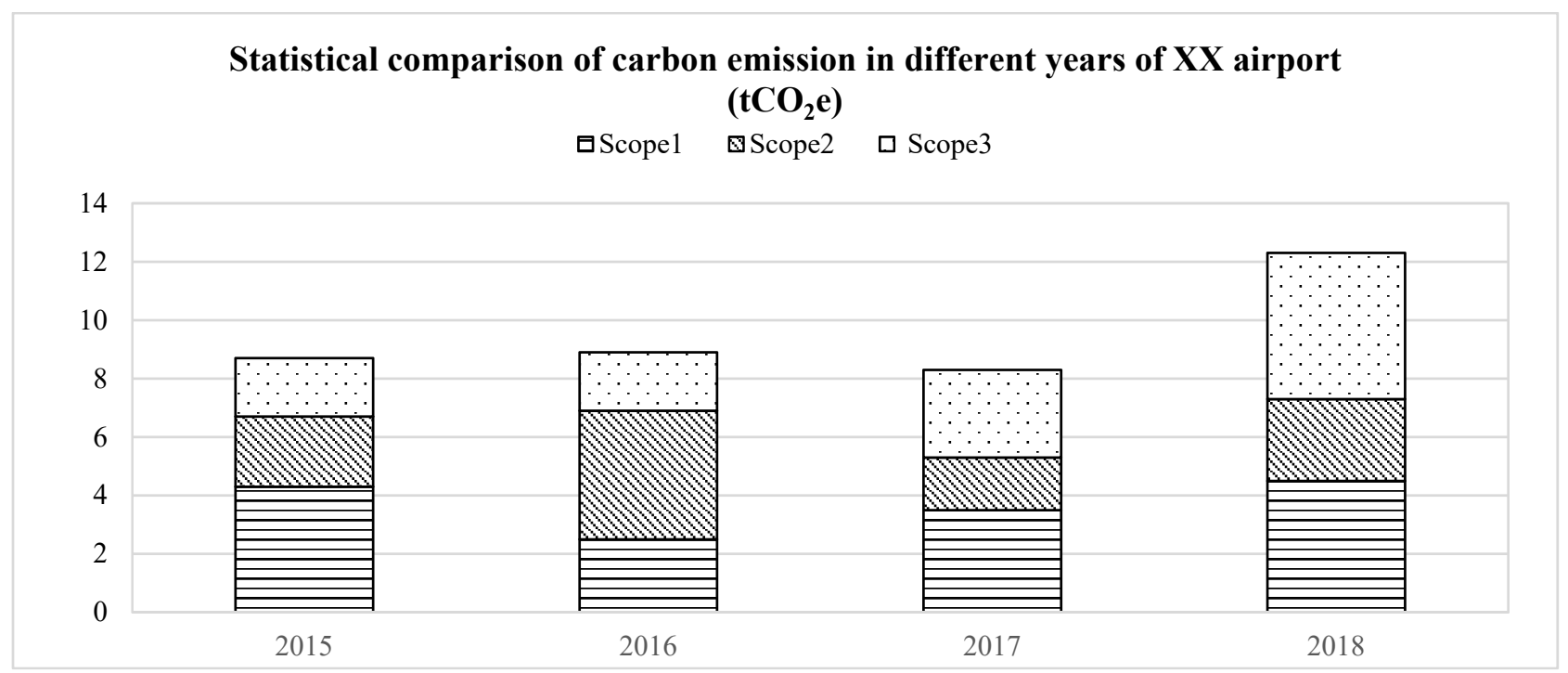

Figure 3. Statistical analysis of carbon emission data

\section{Conclusion}

The carbon emission management software is mainly designed from the perspective of airport energy consumption for serving green airports. Thus, the airport management party can fully grasp the airport carbon emission situation, holistically analyze and compare carbon emission data of airports of different scales and regional climatic conditions based on the industry platform, determine the green level of the airport, and inform the airport management party of the space for airport emission reduction and the direction of improvement to better promote energy conservation and emission reduction. The construction of a green airport is a gradual process and cannot be completed overnight. Therefore, the airport should continue to advance based on the past energy conservation and emission reduction achievements, align short-term interests with long-term development, combine innovative technologies with enhanced management, and integrate green ideas and wisdom.

\section{Acknowledgement}

This work is funded by CAAC Technology Innovation Guidance Program 'Research on Energy Consumption Management of
Civil Airport Terminals' (MHRD20140319).

\section{References}

1. Planning on Energy Conservation and Carbon Reduction of Civil Aviation Industry, CAAC, $(2008,12)$

2. Guidance on Accelerating Energy Conservation and Emission Reduction of Civil Aviation Industry, CAAC, (2011.3)

3. Survey on China Airport Carbon Emission and Countermeasure Study on Emission Reduction, CCAA, 25, (2014)

4. Implementation Opinions on Deepening the Green Development of Civil Aviation, CAAC, (2018.11)

5. Zhu Jialin, Hu Rong, Zhang Junfeng Zheng Lijun, Airport Carbon Emissions Measurement and Time Evolution Characteristics Research, Journal of Wuhan University of Technology(Transportation Science \& Engineering), 43(1), 105,( 2019)

6. Guo Rui,Research on Carbon Emission Computation and Carbon Reduction Technologies for Civil Airports, 12,(2016) 